Background Shoulder dystocia remains a common cause of litigation in obstetrics.

The RCOG Shoulder Dystocia Guideline (2005) recommends auditing all cases of shoulder dystocia to improve training and patient care.

Aim This retrospective cohort study reviewed maternal and fetal complications for all cases of shoulder dystocia from July 1, 2008Dec 31, 2010 at a large University Teaching Hospital in the UK.

Method Cases were collected from the Operating Theatre, Special Care Baby Unit, Delivery Suite and Risk Management Registries.

Results There were 292 cases ( $1 \%$ ) of shoulder dystocia [primigravida ( $\mathrm{n}=100)$, and multiparous $(\mathrm{n}=192)]$. The overall total [Group 1] ( $\mathrm{n}=292$ mean birth-weight $3.979 \mathrm{~kg} \pm 0.475$; the Instrumental Delivery [Group 2] $(\mathrm{n}=94)$ mean birth-weight $3.937 \mathrm{~kg} \pm 0.486$; and the Instrumental Delivery in Theatre [Group $3](n=28)$ mean birth-weight $4.036 \mathrm{~kg} \pm 0.577$. In group 3, a Consultant was present in theatre 19/28 (67.86\%).

\section{FETAL COMPLICATIONS}

SCBU Admission $(\mathrm{n}=17)-5.82 \%$

Macrosomia $>4.5 \mathrm{~kg}(\mathrm{n}=33)-11.30 \%$

Erbs Palsy \& Bone Fracture $(n=6)-2.05 \%$

Stillbirth $(\mathrm{n}=1)$

\section{MATERNAL COMPLICATIONS}

Postpartum Haemorrhage $>1000$ mls $(\mathrm{n}=20)-6.85 \%$

$3^{\text {rd }}$ Degree Tear $(n=22)-7.53 \% \&\left[4^{\text {th }}\right.$ degree Tear $\left.(n=1)\right]$

Severe Shoulder Dystocia

Delivery head-to-body interval $\geq 5$ mins $(n=12)-4.10 \%$

Delivery Required $\geq 3$ Manoeuvres ( $n=34)-11.64 \%$

Conclusion

1. The Risk Management team had a robust proforma with standardise documentation to identify, investigate (Serious Incident Reporting) and include all shoulder dystocia cases in the monthly maternity dashboard.

2. All Erbs palsy/fractures cases had outpatient Paediatric and Physiotherapy followed-up.

3. All staff must attend mandatory training involving shoulder dystocia drills.

\section{PP.64 IMPACT OF ORAL HEALTH OF THE MOTHER DURING PREGNANCY ON ORAL HEALTH OF CHILDREN}

doi:10.1136/archdischild-2013-303966.341

II Bica, 'M Cunha, 'J Costa, ${ }^{2 M}$ Reis Santos. 'Polytechnic Institute of Viseu-Health School-ClaDETS, Viseu, Portugal; '2ICBAS-Porto University, Porto, Portugal

Introduction Dental caries is a major chronic disease in children justifying investigate the effect of oral health of the mother during pregnancy on oral health status of the child. Thus, surveillance of oral health of the mother during pregnancy is essential for the promotion of child health.

Objectives To characterise the oral health of the mother during pregnancy and its effect on oral health status of the children.

Methods Observational and cross study conducted on a random sample of 653 mothers and their respective descendants living in the central region of Portugal.

Material Data collection was conducted through a questionnaire on Oral Health in Pregnancy. It is further preceded to the evaluation index of Decayed, Missing and Filled Teeth (DMFT), through the observation of the oral cavity of the children.

Results Most mothers held health monitoring during pregnancy (84.1\%), while only $72.8 \%$ had six or more appointments, considering that way with a monitored pregnancy The most frequent problems during pregnancy were oral pathology in $18.5 \%$ of mothers, $15.9 \%$ of these showed decayed teeth, $2.4 \%$ gingivitis and $0.3 \%$ periodontitis.

The DMFT of the children varied between 0 and 17 (mean $=2.23$; $\mathrm{SD}=2.484$ ). The children whose mothers had decayed teeth during pregnancy had a higher DMFT index when compared with those whose mothers had no oral problems $(\mathrm{OM}=354.54 \mathrm{vs} \mathrm{OM}=300.40)$, $(\mathrm{U}=20965.5 ; \mathrm{Z}=-2.828 ; \mathrm{p}=0.005)$.

Conclusion The results suggest that on the maternal health consultation and monitoring of pregnancy, health professionals should monitor the oral problems of the pregnant.

\section{PP.65 STUDY OF FACTORS ASSOCIATED WITH LOW BIRTH WEIGHT}

doi:10.1136/archdischild-2013-303966.342

C Chaves, E Coutinho, P Nelas, J Duarte, L Araujo. Polytecnic Institute of Viseu-Superior Health School-Cl\&DETS, Viseu, Portugal

Background Low birth weight is a significant risk factor for neonatal morbidity and mortality as well as a general indicator of the health status of a population. Objectives: To determine the influence of socio-demographic, obstetric and prenatal care variables in low birth weight.

Methods This is a cross-sectional, descriptive, correlational and prospective study. The sample is probabilistic intentional, consisting of 1846 mothers, of which 161 had newborns with low birth weight. A questionnaire was used to collect data between March 2010 and May 2012 in 26 Portuguese public health institutions.

Results In this study, low birth weight was associated to: absence of pathologies during pregnancy, absence of gestational age risk, absence of obstetric risk, maternal age considered of risk or not/ absence of gestational age risk $(p=0.000)$, low education/monthly income level $\leq 1000 €$, low education/unemployment, number of children of risk/absence of gestational age risk, no consumption of alcohol and tobacco/absence of pathologies during pregnancy, no consumption of alcohol and tobacco/absence of gestational age risk pathologies during pregnancy/absence of gestational age risk, presence of previous pathologies/absence of gestational age risk, twin pregnancy/absence of gestational age risk, and with an error of $10 \%$ to low education level, unplanned pregnancy, late onset of prenatal surveillance, and absence of previous pathologies.

Conclusion The determination of the factors that favour the development of low birth weight is of fundamental importance to the understanding, planning and development of actions within mother-child health.

\section{PP.66 AN AUDIT OF REDUCED FETAL MOVEMENTS IN A DISTRICT GENERAL HOSPITAL IN LANARKSHIRE}

doi:10.1136/archdischild-2013-303966.343

LR Kellison, E Ferguson, 0 Wolff. Wishaw General Hospital, Lanarkshire, UK

Background Fetal movements are perceived as a sign of a fetal wellbeing, with reduced fetal movements (RFM) associated with poor perinatal outcome. Antenatal investigation of RFM aims to exclude fetal death and identify pregnancies at risk of adverse outcomes. Wishaw General does not currently have a local RFM guideline.

Aims To audit the management of women presenting to Wishaw General Maternity Unit (WGMU) with RFM, compare with RCOG guidance and devise a local RFM guideline.

Method A retrospective case note review of 23 patients presenting to WGMU.

Results 13 primigravid and 10 parous women, with a mean gestation of 37 weeks (24-41), were included.

Based on KCND 11 were high risk and 12 low risk.

All women received CTG investigation, 100\% were normal. There was no documentation of risk factors for IUFD or of fundal height measurement at presentation.

$4 \%$ of women were booked for USS within 24 hours of presenting with RFM. Induction of labour for RFM occurred in $4 \%$ of patients presenting at term. 